\title{
OPTIMAL STRATEGIES OF A DUAL-CHANNEL GREEN SUPPLY CHAIN WITH RECYCLING UNDER RETAILER PROMOTIONAL EFFORT
}

\author{
BROJESWAR PAL* AND AMIT SARKAR
}

\begin{abstract}
Due to the hugely populated world, recycling of the used products has become the most significant perspective in e-commerce. The scientists have been exploring how increases the degree of recyclability and the green innovation level. This paper considers a supply chain with a manufacturer, a retailer, a supplier, and a collector. The manufacturer can increase or decrease the level of green innovation by changing the quality of raw materials. He sells them through his direct channel as well as the retailer's traditional channel. The retailer enforces the strategy promotional effort for enlarging his market demand. After formulating the problem, the strategies in collector-led, supplier-led, collectorsupplier Nash, and collector-supplier-retailer Nash game are studied under manufacturer Stackelberg games along with the centralized policy. The parameters' sensitivity has been analyzed to the profits and decision variables and then draw significant managerial insights. The model declares the optimal strategies for each player as well as the chain. It is achieved that the higher level of green innovation and promotional effort always increases all the profit. The optimal pricing decisions be lowest under the Collector-Supplier Nash game.
\end{abstract}

Mathematics Subject Classification. 90B06.

Received October 1, 2020. Accepted January 25, 2021.

\section{INTRODUCTION}

\subsection{Motivation}

Green innovation (GI) is a notion of managing the environment to reduce environmental pollution such as urban sprawl, non-sustainable resource consumption, industrial and household waste, etc. European Union sets a target to recycle at least $70 \%$ of all packaged goods by 2030 and for household recycling rates to be $65 \%$ by 2035 (https://www.nspackaging.com/analysis/best-recycling-countries/). To increase the recycling rate, one can take small steps like using biodegradable plastics while packaging and using more eco-friendly materials to produce household products. In the recycling process, used products are pulled together from the customers in several ways, and the manufacturer receives the recyclable wastes. This type of supply chain (SCN) is called a closed-loop (CLP) SCN. In this article, the collector collects the used products which are recyclable and then deliver to the manufacturer. To avoid the shortfall of raw material, the manufacturer may have brought fresh raw materials from the supplier [29].

Keywords. Green supply chain management, green innovation, closed-loop dual channel, promotional effort, recycling.

Department of Mathematics, The University of Burdwan, Burdwan 713104, India.

*Corresponding author: brojo_math@yahoo.co.in 
Again, it may be profitable to make some agreement among the members. For instance, many reputed companies, like Dell, Adidas, etc., cooperate with their respective raw material suppliers, and also soft drinks companies deal with some third party suppliers [10]. In this study, two types of strategies have been considered, such as Centralized policy (CP) and Manufacturer Stackelberg (MS) game. All the possible subcases have been studied under the MS game. In the commercial world, promotional effort (PE) is an essential useful tool for the retailer's standpoint. The effort is given to the customers by advertising, free sample show, free installation, sponsoring, and explanation of using methods [3]. For instance, if a retailer explains the basic features, offers installation free services, shows a free practical demonstration, customers automatically become biased to buy it from that retailer.

\subsection{Novelties}

The recent articles with CL SCN have been listed in Table 1. In the present article, we consider strategies: $\mathrm{CP}$, three different strategies under MS, NG. Some of the strategies have been considered for the first time under this model formulation. In recent trends, recycling of the used products becomes an important aspect for the environment and, consequently, for the customers. So it is very exigent to include the collector of the waste as a member of the SCN and recycle the used products. Our present study focuses on optimizing the players' profits and the total profit (TPT) by using some specific game-theoretic approaches under the DC CLP green SCN. The parameters' sensitivity against the profit functions and the decision variables are analyzed, and then managerial insights are obtained.

The remaining paper is oriented as follows:

some related literature has been reviewed in Section 2. Then the notations are listed, and the assumptions of the model are defined in Section 3. Section 4 framed the model, and the profit functions are obtained under different strategies. In Section 5, a numerical example is constructed and analyzed the outcomes. Then the sensitivity of some of the parameters is analyzed in Section 6. After that, managerial insights have been discussed in the Section 7 . Then the overall conclusion of the study is drawn in Section 8.

\section{Literature REVIEW}

In this section, the literature related to our research directions are discussed such as: Dual channel (DC) supply chain (SCN) with recycling, GI and PE.

\subsection{Dual channel supply chain with recycling}

Due to the rapid increment of population and short life-cycled products, the wastes have increased dramatically in recent times. Many first economic countries like Germany, has implemented various strategies to increase the rate of recycling. The useful waste materials of the households and industries have recycled and then used to produce new materials [2,28]. Then, Giovanni [9] have characterized a two-echelon (2En) CLP SCN in the battery industry and formulated a model with considering a manufacturer-retailer duality under MS game with recycling, where they have found that a maximum joint incentive always increases the GI level and profit of the manufacturer. After that, Shi et al. [25] has constructed a stylized DC CLP SCN with one manufacturer, one retailer, and one recycler. They have considered the manufacturer as a leader and then optimize the effectiveness and competitiveness by incorporating one of the remaining entities. The main findings of the study are that it has connected the business practices and theoretical explorations. Yuan et al. [34] has adopted useful method of optimization analysis and game theory to analyze for the players to achieve interest coordination under equilibrium states. They have also demonstrated the strength of their obtained results by numerical analysis.

\subsection{Green innovation}

In the study [33], a 2En SCN has been considered where a supplier sells through a retailer. The supplier is liable to impose GI in the materials that reduce the unit production cost and the harmfulness on the environment. 
OPTIMAL STRATEGIES OF A DUAL-CHANNEL GREEN SUPPLY CHAIN

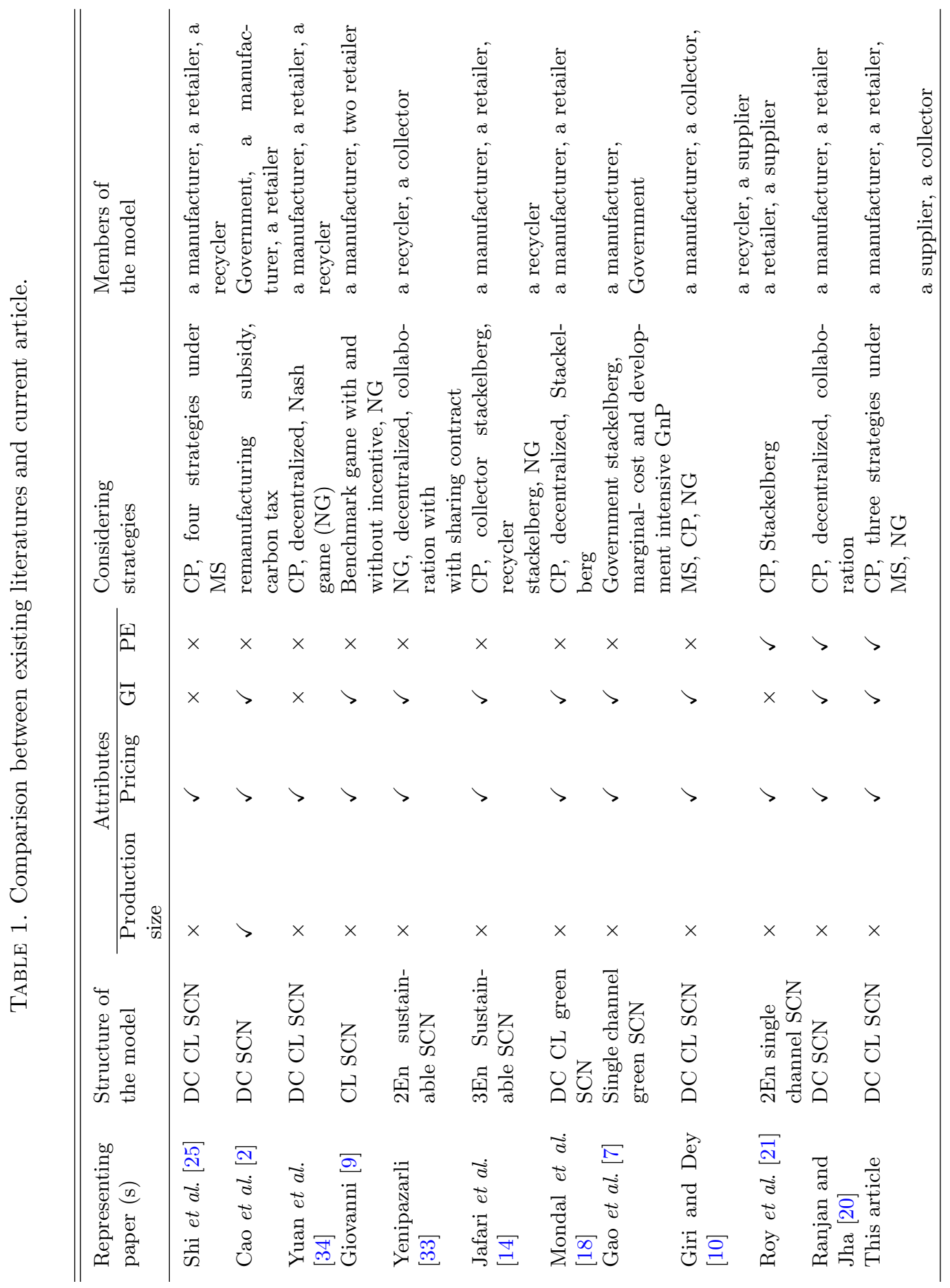


Then they have studied the profit for upstream eco-efficient innovation under CP. Jafari et al. [14] has studied the economic and environmental perspectives under a 3En DC SCN with one collector, one manufacturer, and one recycler. They have found that the manufacturer opted for the collector when some thresholds are met. They have also determined the optimal strategy for the manufacturer under various games. A SCN has been considered by Gao et al. [7] in which a manufacturer can produce a green product (GnP), and the GI level has been determined by the manufacturer only if the GI level does not violate the green standard of government rule. They have also analyzed the sensitivity between $\mathrm{GnP}$ and non-GnP. The article Mondal et al. [18] have considered a green SCN where the manufacturer can produce and sell GnP through the online and traditional retail channel. The manufacturer collects the used products from the customers to recycle them. Then the susceptibility of the assumed parameters on the optimal decisions has been analyzed. Also, they have obtained important results with the assistance of a numerical example. Xie et al. [30] has studied the effect of green subsidies on the relationship between GI and financial performance. They have also drawn some useful managerial insights with the support of observations. Wang and Song [27] has investigated the pricing policies for the DC SCN within the presence of GI and PE. They have also considered that the manufacturer can produce two kinds of products: GnP, and non-GnP. The optimal decisions have been compared under three models and implemented the sensitivity analysis of the parameters. After that, the article Karim and Nakade [16] has considered a SCN model with a retailer and a manufacturer under demand disruption. This paper aims to optimize the production, green investment, and the resourcing decisions of the product so that the players can grab the maximum profit. They have concluded that the rate of disruption of the products affects all the decision variables.

\subsection{Promotional effort}

The retailer gives this effort to the customers to prompt his/her products. Advertising, public relations, free sample show, direct response marketing, sponsoring, social media, explanation of the guidelines of uses are examples of the PE $[4,6]$. Roy et al. [21] has considered a 2En SCN with one supplier and one retailer where the demand rate is uncertain and depending on PE. All the profit functions of the proposed model have been analyzed and also compared them under different strategies. The pricing and coordination strategies have been studied by Ranjan and Jha [20] for a DC SCN. Here, the manufacturer can produce GnP and non-GnP and sell them to the retailer who provides the sales effort of the non-green-products. They have also developed a Stackelberg game-theoretic approach and then obtained the equilibrium results. After that, a 2En SCN has been considered by Yang et al. [32] consisting of a supplier and a retailer. They have identified the effects of the advertising elasticity and the interest rate on the pricing decisions. Also, numerical examples have been studied and analyzed the sensitivity of the parameters of the model. He and Zhou [12] has proposed a DC green SCN consisting of a manufacturer and a retailer. To prompt himself, the retailer gives more effort to sell products, which are called PE. They have examined the performance of the SCN under PE and analyzed the sensitivity of the PE parameters.

Besides these, many of the researchers have worked in these directions in recent trends [8,23,31]. From the Table 1, it can be concluded that this study is partially similar to Ranjan and Jha [20] and Mondal et al. [18]. But in this study, DC CLP green SCN has been considered with promotional effort. In the previous study, no researchers have considered such type of model.

\section{Problem description}

In this section, the problem is described at first and secondly listed down the assumptions and defines the used notation. Due to the enormous population and using eco-harmful products, recycling the used products has become crucial. On behalf of this, the present study aims to collect the recyclable used products and refurbish them accordingly. A 2En DC CLP SCN is formulated with a manufacturer, a collector, a supplier, and a retailer. Now, the question is, how be the raw materials collected? The collector gathers the waste (i.e., used products) from the customers and sells them to the manufacturer. Then, the manufacturer converts this waste into fresh 
raw materials. The rest of the raw materials are brought from the suppliers to make sure that they can produce enough finished GnP to soothe the customers' demand. The manufacturer produces the items for customers and sells them through the retailer or his online channel. Then the optimal pricing decisions and the Level of GI and PE are computed under different strategies like CP, NG and three types of decision making under MS.

\subsection{Notation}

The following notations have been used throughout the paper:

\begin{tabular}{|c|c|}
\hline & Parameters \\
\hline$c_{c}$ & Unit collection cost of recyclable wastes to the collector $(\$)$. \\
\hline$\phi$ & $\begin{array}{l}\text { Recyclability degree of waste denoting the portion of waste }(0<\phi<1) \\
\text { that can be recovered and turned into new product. }\end{array}$ \\
\hline$\gamma$ & Number of finished product by one unit of raw material $(0<\gamma<1)$. \\
\hline$\beta$ & Fractional part of recyclable product of one unit used product $(0<\beta<1)$. \\
\hline$a_{1}, b_{1}(>0)$ & $\begin{array}{l}\text { Maximum possible demand faced by the manufacturer for the finished } \\
\text { product in online channel and traditional channel. }\end{array}$ \\
\hline$a_{2}, a_{3}, b_{2}(>0)$ & Price sensitivity of the customers demand. \\
\hline$a_{4}(>0)$ & Sensitivity of the PE on the customers demand. \\
\hline$a_{5}, b_{5}(>0)$ & $\begin{array}{l}\text { Positive and negative effects of the GIs } \\
\text { on the customers' demand respectively. }\end{array}$ \\
\hline$p_{\text {so }}$ & Cost of the new raw material of the supplier $(\$)$. \\
\hline \multirow[t]{2}{*}{$p_{r c}$} & Cost to recycle the waste collected from the collector. \\
\hline & Decision variables \\
\hline$p_{d}$ & Price charged by the collector for one unit of recyclable waste $(\$)$. \\
\hline$p_{2}$ & $\begin{array}{l}\text { Price charged by the manufacturer to the customers for one unit of } \\
\text { finished product in online channel }(\$) \text {. }\end{array}$ \\
\hline$p_{m}$ & $\begin{array}{l}\text { Price charged by the manufacturer to the retailer for one unit of } \\
\text { finished product in indirect channel }(\$) \text {. }\end{array}$ \\
\hline$p_{1}$ & $\begin{array}{l}\text { Price charged by the retailer to the customers for one unit of } \\
\text { finished product in indirect channel }(\$) \text {. }\end{array}$ \\
\hline$p_{s}$ & Price charged by the supplier for one unit of fresh raw material $(\$)$. \\
\hline$s$ & Level of promotional effort. \\
\hline \multirow[t]{2}{*}{$\theta$} & GI level $\left(0<\theta<\theta_{\max }\right)$. \\
\hline & Dependent variables \\
\hline$D_{1}$ & Customer demand of the manufacturer in online channel. \\
\hline$D_{2}$ & Customer demand of the manufacturer in traditional channel. \\
\hline$D_{r c}$ & Manufacturers demand of recyclable wastes meet up by the collector. \\
\hline$D_{s}$ & Manufacturers demand of fresh raw materials meet up by the supplier. \\
\hline
\end{tabular}

\subsection{Assumptions}

The assumptions which are made in this article, are listed below:

- The collector collects the used recyclable waste from the customers at a price $c_{c}$ and sells it to the manufacturer at $p_{d}$.

- The manufacturer has the ability to enlarge or reduce the GI level of produced products accordingly. Here, we use a scale 0 to 100 to measure the level of GI, where 100 means the product is $100 \%$ green innovative, and it can be recycled completely, and 0 means these kinds of products has $0 \%$ recyclable parts.

- The retailer buys products from the manufacturer and sells it to the customers through the traditional channel. The retailer does not allow shortage and as well as does not stock. To exist in the comparative markets, the retailer gives some effort to promote the products.

- When the decision power of a member is greater than the other members, he expects his profit value would be greater than others' profit values. In this case, when a member acts as the leader, he admits to contributing 
to the supply chain if the proportion of his profit and his followers' profits is more than a constant value. For example, when the retailer has more decision powers than the collector, then the retailer contributes to the supply chain if, $\frac{\Pi_{r}}{\Pi_{c}}>\tau_{r}$ where $\tau_{r}>1$. For similar reason, we take $\tau_{s}$ and so for $\tau_{c}$.

- In the model, we consider that the customers' demand depends linearly on the pricing of the products, the service, and the GI level of the products $[5,13]$. It is the most simple form of demand, and most of the researchers like to choose such types. Next, the demand is monotonically decreasing with its own channel pricing and monotonically increasing with cross-channel pricing. It is also assumed that GI and PE positively affect the customers' purchasing rate and so on the demand. Therefore, the demand rates of the channels are as follows:

$$
\begin{gathered}
\text { Retail channel: } D_{1}=a_{1}-a_{2} p_{1}+a_{3} p_{2}+a_{4} \theta+a_{5} s, \\
\text { Direct channel: } D_{2}=b_{1}-b_{2} p_{2}+a_{3} p_{1}+a_{4} \theta-b_{5} s .
\end{gathered}
$$

Therefore the total demand by the customer is $D_{1}+D_{2}=D$ (say) ( $D_{1}$ and $D_{2}$ are taken from (3.1) and (3.2)). All the associated parameters of the demand rates are described in the Notation Section 3.1.

- Next, a discount policy is considered to improve the relationship between the manufacturer and the retailer. We assume that the manufacturer will provide a discount $\left(k_{1}\right)$ on the online price to the retailer as the retailer brought a large quantity of the products at a time, i.e., $p_{m}=k_{1} p_{2}$ and the production cost of the manufacturer is assumed negligible.

- In recent trends, due to the accessibility of online channels is greater than the retail channel from the customers' point of view. Therefore, The market potential of direct channel is assumed to be greater than the traditional channel (i.e., $b_{1}>a_{1}$ ). Also, the own-channel pricing sensitivity is higher than the crosschannel pricing sensitivity on the demand rates (i.e., $a_{2}>a_{3}$ and $b_{2}>b_{3}$ ) [17].

- The total possible collected recyclable products are $\beta$ times of total used product $D$. Therefore, the demand of the collector is $D_{r c}=\beta D$. Here, $D$ is the number of finished products is produced from $\gamma D$ raw materials.

- From the collected recyclable products, the manufacturer recycles $\phi \beta D$ number of raw materials, and the supplier deals rest of the raw materials. Therefore the demand of the supplier is $D_{s}=(\gamma D-\beta \phi D)$, where $\beta=\rho_{1} \frac{\theta}{\theta_{\max }}<1$ and $\rho_{1}>0$ is constant. The value of the "fractional part of the recyclable product of one unit used product" directly depends on the GI level of the respective product.

- GI does not hamper the marginal cost of the manufacturer $[1,26]$ but, to produce GnP, the manufacturer should spend some extra to achieve the GI level. Here the cost function of GI level is considered as $\frac{1}{2} \xi \theta^{2}$ [17]. Also, the cost of offering PE is $\frac{1}{2} \eta s^{2}$ [3]. In general, the marketing executive (ME) measures the volume of the PE by observing PE's effectiveness on the customers' minds. As a result, the ME and the selling strategies' efficacy are the main goals for scaling PE. So that PE has no specific unit.

\section{Model FORMUlation}

In this section, the profit functions of the members of the proposed model are derived. Then three types of strategies are considered, such as CP, MS, and NG. Then the solutions for decision variables are derived for each strategy. The diagram of the proposed model can be understood in Figure 1.

To calculate the profit functions of the members, first, calculate the revenue and then the cost functions for each individual. Then, the revenue of the manufacturer is $=$ total demand in the retail channel $\times$ wholesale price + total demand in online channel $\times$ direct channel price $=p_{1} D_{1}+p_{2} D_{2}$, and the manufacturer's total cost is $=$ cost of purchasing recyclable waste from collector + cost of purchasing row materials from supplier + cost of imposing GI + cost of recycle the waste $=D_{r c} \times p_{d}+D_{s} \times p_{s}+\frac{1}{2} \xi \theta^{2}-p_{r c} D_{r c}$. Therefore, the profit of the manufacturer is $=$ the revenue of the manufacturer - the manufacturer's total cost $=p_{2} D_{2}+D_{1} p_{m}-\left(D_{r c} p_{d}+\right.$ $\left.D_{s} p_{s}+\frac{1}{2} \xi \theta^{2}+p_{r c} D_{r c}\right)$. Next, the revenue of the retailer is $=$ the total demand in the retail channel $\times$ retail price $=p_{1} \times D_{1}$, and the retailer's cost is $=$ products brought from the manufacturer $\times$ wholesale price + cost of giving promotional effort $=p_{m} \times D_{1}+\frac{1}{2} \eta s^{2}$. Hence, the TPT of the retailer is $=$ total revenue - total cost $=$ $p_{1} D_{1}-p_{m} D_{1}-\frac{1}{2} \eta s^{2}$. Consequently, the TPT of the collector is $=$ total revenue - total cost $=p_{d} D_{r c}-c_{c} D_{r c}$, 


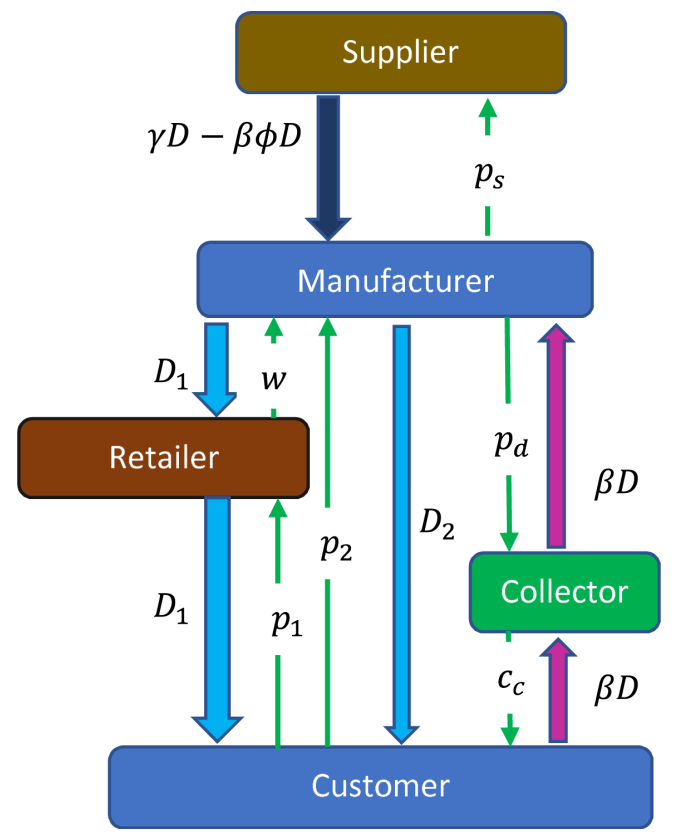

Figure 1. Graphical representation of the model. Descriptions of the symbol are given in Section 3.1.

and the profit of the supplier is $=p_{s} D_{s}-p_{s o} D_{s}$. Therefore, the profit functions of the manufacturer, retailer, collector and the supplier are given by

$$
\begin{aligned}
\Pi_{m} & =p_{2} D_{2}+D_{1} p_{m}-\left(D_{r c} p_{d}+D_{s} p_{s}+\frac{1}{2} \xi \theta^{2}\right)-p_{r c} D_{r c} \\
\Pi_{r} & =\left(p_{1}-p_{m}\right) D_{1}-\frac{1}{2} \eta s^{2} \\
\Pi_{c} & =\left(p_{d}-c_{c}\right) D_{r c} \\
\Pi_{s} & =\left(p_{s}-p_{s o}\right) D_{s}
\end{aligned}
$$

respectively, where the profits of manufacturer, retailer, collector and supplier are donated by subscripting $m$, $r, c$ and $s$.

\subsection{Centralized policy}

The CP is a scheme where all the players act collectively, i.e., there does no exist neither follower nor leader. Therefore, the total channel profit $\left(\Pi_{T}\right)$ is given by

$$
\begin{aligned}
\Pi_{T} & =\Pi_{m}+\Pi_{r}+\Pi_{c}+\Pi_{s} \\
& =p_{1} D_{1}+p_{2} D_{2}-c_{c} D_{r c}-p_{s 0} D_{s}-\frac{1}{2} \xi \theta^{2}-\frac{1}{2} \eta s^{2}-p_{r c} D_{r c} .
\end{aligned}
$$

Now the optimal level of pricing, green level, and PEs are discussed of the proposed model under the CP. 


\subsubsection{Solution procedure}

To obtain the maximum profit for the $\mathrm{CP}$, at first the first order conditions of (4.5) is solved, i.e., we have to solve the following system of equations:

$$
\begin{aligned}
& \left\{\begin{array}{l}
\frac{\partial \Pi_{T}}{\partial p_{1}}=0 \\
\frac{\partial \Pi_{T}}{\partial p_{2}}=0 \\
\frac{\partial \Pi_{T}}{\partial \theta}=0 \\
\frac{\partial \Pi_{T}}{\partial s}=0
\end{array}\right. \\
& \left\{\begin{array}{l}
a_{1}-2 a_{2} p_{1}+2 a_{3} p_{2}+a_{5} s+a_{4} \theta+a_{2} p_{s 0} \gamma-a_{3} p_{s 0} \gamma+\left(a_{2}-a_{3}\right) \theta\left(c_{c}+p_{r c}-p_{s 0} \phi\right) \rho_{1}^{\prime}=0 \\
b_{1}+2 a_{3} p_{1}-2 b_{2} p_{2}-b_{5} s+a_{4} \theta-a 3 p_{s 0} \gamma+b_{2} p_{s 0} \gamma-\left(a_{3}-b_{2}\right) \theta\left(c_{c}+p_{r c}-p_{s 0} \phi\right) \rho_{1}^{\prime}=0 \\
a_{4} p_{1}+a_{4} p_{2}-\theta \xi-2 a_{4} p_{s 0} \gamma-2 a_{4} \theta\left(c_{c}+p_{r c}-p_{s 0} \phi\right) \rho_{1}^{\prime} \\
-\left(a_{1}+b_{1}-a_{2} p_{1}+a_{3} p_{1}+a_{3} p_{2}-b_{2} p_{2}+a_{5} s-b_{5} s+2 a_{4} \theta\right)\left(c_{c}+p_{r c}-p_{s 0} \phi\right) \rho_{1}^{\prime}=0 \\
-b_{5} p_{2}-s \eta+b_{5} p_{s 0} \gamma+a_{5}\left(p_{1}-p_{s 0} \gamma\right)-\left(a_{5}-b_{5}\right) \theta\left(c_{c}+p_{r c}-p_{s 0} \phi\right) \rho_{1}^{\prime}=0, \text { where } \rho_{1}^{\prime}=\frac{\rho_{1}}{\theta_{\max }} .
\end{array}\right.
\end{aligned}
$$

Solving the above equations, we get $p_{1}^{*}, p_{2}^{*}, \theta^{*}$, and $s^{*}$. If the Hessian matrix (HM) of the profit function (4.5) is negative definite, then $p_{1}^{*}, p_{2}^{*}, \theta^{*}$, and $s^{*}$ will be the optimal value of the decision parameters $p_{1}, p_{2}, \theta$ and $s$ respectively.

\subsection{Manufacturer Stackelberg game}

In this section, the manufacturer acts as a leader, and the other players (retailer, collector, and the supplier) follow the leader. Therefore, the manufacturer sets all the pricing decisions first, and the followers also decide their pricing decisions according to their decision power. Also, it is considered that the retailer has the next most power to decision making after the manufacturer. Based on the ability of decision making of the collector and supplier, three gaming plans are considered such as collector-led game (CL game), supplier-led game (SL game), and collector-supplier Nash (CS Nash). We also analyze the NG model among the retailer, supplier, and collector under MS game.

\subsubsection{Collector-led game}

In this model, the collector has more decision power than the supplier. So, the collector fixes his price, and then the supplier sets his own accordingly. Then the formulation of the model is as follows:

$$
\left\{\begin{array}{l}
L_{1}: \max \Pi_{m}=p_{2} D_{2}+D_{1} p m-\left(D_{r c} p_{d}+D_{s} p_{s}+\frac{1}{2} \xi \theta^{2}\right)-p_{r c} D_{r c} \\
L_{2}: \max \Pi_{r}=\left(p_{1}-p_{m}\right) D_{1}-\frac{1}{2} \eta s^{2} \\
L_{3}: \max \Pi_{c}=\left(p_{d}-c_{c}\right) D_{r c} \\
L_{4}: \max \Pi_{s}=\left(p_{s}-p_{s o}\right) D_{s} .
\end{array}\right.
$$

Now, using the equation (4.6) and setting $\frac{\Pi_{r}}{\Pi_{c}} \geq \tau_{r}$, we get $p_{d} \leq \mathfrak{F}_{1}\left(p_{1}, p_{2}, s, \theta, p_{s} ; \tau_{r}\right)$. Since $\Pi_{c}$ is linearly increasing on $p_{d}$, the optimal value of $p_{d}$ is the upper bound of $p_{d}$. Therefore we set

$$
p_{d}=\mathfrak{F}_{1}\left(p_{1}, p_{2}, s, \theta, p_{s} ; \tau_{r}\right) .
$$

By similar procedure, setting $\frac{\Pi_{c}}{\Pi_{s}} \geq \tau_{c}$, the optimal value of $p_{s}$ can be decided by supplier as

$$
p_{s}=\mathfrak{F}_{2}\left(p_{1}, p_{2}, s, \theta ; \tau_{c}\right) .
$$

Now, substituting (4.8) in (4.7), we get

$$
p_{d}=\mathfrak{F}_{1}^{\prime}\left(p_{1}, p_{2}, s, \theta ; \tau_{c}, \tau_{r}\right)
$$


Then, We partially differentiate the profit function of the retailer $\Pi_{r}$ with respect to $p_{1}$ and $s$. Then, by equating the first order derivative to zero and by solving, we get

$$
\left\{\begin{array}{l}
p_{1}=\mathfrak{F}_{3}\left(p_{2}, \theta ; \tau_{c}, \tau_{r}\right) \\
s=\mathfrak{F}_{4}\left(p_{2}, \theta ; \tau_{c}, \tau_{r}\right) .
\end{array}\right.
$$

These values are optimal if the HM of the profit function is negative definite. In this case, for the complexity, The negative definiteness of the HM of the profit function can be checked numerically as expressions. Using (4.8)-(4.10), we transfer the profit function of manufacturer $\Pi_{m}$ to a function of $p_{2}$ and $\theta$. Then the function is optimized with respect to $p_{2}$ and $\theta$ and get constant values $p_{2}^{*}$ and $\theta^{*}$ respectively. Similarly, Using these values, we obtain the optimal values of $p_{r}, p_{d}$ and $p_{s}$ as $p_{r}^{*}, p_{d}^{*}$ and $p_{s}^{*}$ respectively from (4.8) to (4.10).

\subsubsection{Supplier-led game}

In this model, the supplier has more decision power than the collector. So, the supplier determines his price, and then the collector adjusts his own accordingly. The model is formulated as follows:

$$
\left\{\begin{array}{l}
L_{1}: \max \Pi_{m}=p_{2} D_{2}+D_{1} p m-\left(D_{r c} p_{d}+D_{s} p_{s}+\frac{1}{2} \xi \theta^{2}+p_{r c} D_{r c}\right) \\
L_{2}: \max \Pi_{r}=\left(p_{1}-p_{m}\right) D_{1}-\frac{1}{2} \eta s^{2} \\
L_{3}: \max \Pi_{s}=\left(p_{s}-p_{s o}\right) D_{s} \\
L_{4}: \max \Pi_{c}=\left(p_{d}-c_{c}\right) D_{r c} .
\end{array}\right.
$$

Using $\frac{\Pi_{r}}{\Pi_{s}} \geq \tau_{r}$, and then making some calculation, we obtain $p_{s} \leq \mathfrak{G}_{1}\left(p_{1}, p_{2}, s, \theta, p_{d} ; \tau_{r}\right)$. Since the profit of the supplier $\Pi_{s}$ linearly increasing in $p_{s}$, the upper bound is the optimal value of $p_{s}$. Therefore,

$$
p_{s}=\mathfrak{G}_{1}\left(p_{1}, p_{2}, s, \theta, p_{d} ; \tau_{r}\right) .
$$

Similarly, using $\frac{\Pi_{s}}{\Pi_{c}} \geq \tau_{s}$ and (4.12), we have $p_{d} \leq \mathfrak{G}_{2}\left(p_{1}, p_{2}, s, \theta ; \tau_{r}, \tau_{s}\right)$. As $\Pi_{c}$ is linearly increasing in $p_{d}$, the optimal value of $p_{d}$ is given by

$$
p_{d}=\mathfrak{G}_{\perp}\left(p_{1}, p_{2}, s, \theta ; \tau_{r}, \tau_{s}\right) .
$$

Substituting (4.13) on (4.12), we get

$$
p_{s}=\mathfrak{G}_{1}^{\prime}\left(p_{1}, p_{2}, s, \theta ; \tau_{r}, \tau_{s}\right) .
$$

After that, the profit of the retailer $\Pi_{r}$ is optimized with respect to $p_{1}$ and $s$, the optimal values can be obtained as follows:

$$
\left\{\begin{array}{l}
p_{1}^{*}=\mathfrak{G}_{3}\left(p_{2}, \theta ; \tau_{r}, \tau_{s}\right) \\
s^{*}=\mathfrak{G}_{4}\left(p_{2}, \theta ; \tau_{r}, \tau_{s}\right)
\end{array}\right.
$$

respectively. Using (4.13)-(4.15), we also optimize the profit of the manufacturer $\Pi_{m}$ with respect to $p_{2}$ and $\theta$ and get the optimal values of them as $p_{2}^{*}$ and $\theta^{*}$ respectively. Using the values of $p_{2}^{*}$ and $\theta^{*}$, the optimal values $p_{1}^{*}, s^{*}, p_{d}^{*}$ and $p_{s}^{*}$ can be obtained.

\subsubsection{Collector-Supplier Nash}

Here, let us consider that the collector and supplier have equal decision power. Therefore, both of them fix their prices independently and simultaneously only after the manufacturer and the retailer. The model is formulated as follows:

$$
\left\{\begin{array}{l}
L_{1}: \max \Pi_{m}=p_{2} D_{2}+D_{1} p m-\left(D_{r c} p_{d}+D_{s} p_{s}+\frac{1}{2} \xi \theta^{2}\right)-p_{r c} D_{r c} \\
L_{2}: \max \Pi_{r}=\left(p_{1}-p_{m}\right) D_{1}-\frac{1}{2} \eta s^{2} \\
L_{3}: \max \Pi_{s}=\left(p_{s}-p_{s o}\right) D_{s} \\
L_{3}: \max \Pi_{c}=\left(p_{d}-c_{c}\right) D_{r c} .
\end{array}\right.
$$


Since $\Pi_{c}$ is linearly increasing in $p_{d}$, it can be argued similarly to the previous game models to get the optimal price of the collector. The optimal price of $p_{d}$ is given by

$$
p_{d}=\mathfrak{H}_{\mathbf{1}}^{\prime}\left(p_{1}, p_{2}, s, \theta, p_{s} ; \tau_{r}\right) .
$$

Similarly, the optimal price of the supplier can be obtained as

$$
p_{s}=\mathfrak{H}_{2}^{\prime}\left(p_{1}, p_{2}, s, \theta, p_{d} ; \tau_{r}\right) .
$$

From (4.17), the value value of $p_{s}$ can be obtained. Let

$$
p_{s}=\mathfrak{H}_{2}^{\prime \prime}\left(p_{1}, p_{2}, s, \theta, p_{d} ; \tau_{r}\right) .
$$

Equating the right hand side of (4.18) and (4.19), we have

$$
p_{d}=\mathfrak{H}_{1}^{\prime \prime}\left(p_{1}, p_{2}, s, \theta ; \tau_{r}\right) .
$$

Now from (4.18) and (4.20), we have

$$
p_{s}=\mathfrak{H}_{2}\left(p_{1}, p_{2}, s, \theta ; \tau_{r}\right) .
$$

Then using this value of $p_{s}$, we can obtain

$$
p_{d}=\mathfrak{H}_{1}\left(p_{1}, p_{2}, s, \theta ; \tau_{r}\right) .
$$

Then using the values of $p_{d}$ and $p_{s}$ from (4.21) and (4.22), the optimal values of $p_{1}, p_{2}, s$ and $\theta$ can be obtained in similar method as previous models.

\subsubsection{Nash game}

In this model, all the players except the manufacturer have the same decision power and decide independently and simultaneously. The proposed model is formulated below:

$$
\left\{\begin{array}{l}
L_{1}: \max \Pi_{m}=p_{2} D_{2}+D_{1} p_{m}-\left(D_{r c} p_{d}+D_{s} p_{s}+\frac{1}{2} \xi \theta^{2}+p_{r c} D_{r c}\right) \\
L_{2}: \max \Pi_{r}=\left(p_{1}-p_{m}\right) D_{1}-\frac{1}{2} \eta s^{2} \\
L_{2}: \max \Pi_{s}=\left(p_{s}-p_{s o}\right) D_{s} \\
L_{2}: \max \Pi_{c}=\left(p_{d}-c_{c}\right) D_{r c} .
\end{array}\right.
$$

As the previous model, using $\frac{\Pi_{m}}{\Pi_{s}} \geq \tau_{m}$ and $\frac{\Pi_{m}}{\Pi_{c}} \geq \tau_{m}$ and with some calculation, the following inequalities can be obtained as $p_{s} \leq \mathfrak{K}_{1}\left(p_{1}, p_{2}, s, \theta, p_{d} ; \tau_{m}\right)$ and $p_{d} \leq \mathfrak{K}_{2}\left(p_{1}, p_{2}, s, \theta, p_{s} ; \tau_{m}\right)$ respectively. Since $\Pi_{s}$ and $\Pi_{c}$ are linearly increasing with $p_{s}$ and $p_{d}$ respectively, so the optimal values of $p_{s}$ and $p_{d}$ exist at the upper bounds. Therefore, the optimal values are as follows:

$$
\begin{aligned}
& p_{s}=\mathfrak{K}_{1}\left(p_{1}, p_{2}, s, \theta, p_{d} ; \tau_{m}\right) \\
& p_{d}=\mathfrak{K}_{2}^{\prime}\left(p_{1}, p_{2}, s, \theta, p_{s} ; \tau_{m}\right) .
\end{aligned}
$$

Now, we solve the first order condition of the profit function $\Pi_{r}$ with respect to $p_{1}$ and $s$. Let the solutions are $p_{1}=p_{1}^{*}$ and $s=s^{*}$. If the HM of the profit function $\Pi_{r}$ is negative definite at $p_{1}^{*}, s^{*}$ then the function is maximum at that point and the values, $p_{1}^{*}$ and $s^{*}$ are optimal. Then putting all the optimal values of the decision variables $p_{1}, s, p_{d}$ and $p_{s}$ on the profit function $\Pi_{m}$, we get $\Pi_{m}^{*}$. Then the first-order conditions of $\Pi_{m}^{*}$ for $p_{2}$ and $\theta$ is solved and getting their values as $p_{2}^{*}$ and $\theta^{*}$ respectively. If the HM of the profit function $\Pi_{m}^{*}$ is negative definite, then $p_{2}^{*}$ and $s^{*}$ are the optimal values of $p_{2}$ and $s$ respectively. Then the optimal profits of the collector, the retailer, and the manufacturer can be obtained from equation (4.23). 
TABLE 2. Optimal values of decision variables under different game models.

\begin{tabular}{llllll}
\hline \hline $\begin{array}{l}\text { Optimal } \\
\text { results }\end{array}$ & $\begin{array}{l}\text { CP } \\
(\$)\end{array}$ & $\begin{array}{l}\text { CL } \\
\text { game (\$) }\end{array}$ & $\begin{array}{l}\text { SL } \\
\text { game (\$) }\end{array}$ & $\begin{array}{l}\text { CS } \\
\text { Nash }(\$)\end{array}$ & $\begin{array}{l}\text { NG } \\
(\$)\end{array}$ \\
\hline$p_{1}^{*}$ & 136.18 & 124.408 & 104.40 & 103.18 & 112.01 \\
$p_{2}^{*}$ & 132.54 & 113.24 & 99.38 & 98.89 & 102.44 \\
$\theta^{*}$ & 95.87 & 56.2634 & 28.63 & 26.42 & 42.45 \\
$s^{*}$ & 9.76193 & 5.63591 & 4.15 & 4.01 & 5.01 \\
$p_{d}$ & - & 14.0468 & 29.30 & 40.7599 & 32.39 \\
$p_{s}$ & - & 26.1083 & 32.84 & 32.5578 & 35.12 \\
$\Pi_{m}^{*}$ & - & 14050.9 & 8173.24 & 7430.86 & 5977.45 \\
$\Pi_{r}^{*}$ & - & 5526.85 & 2994.77 & 2799.17 & 4365.51 \\
$\Pi_{c}^{*}$ & - & 921.14 & 1535.78 & 2153.21 & 3415.69 \\
$\Pi_{s}^{*}$ & - & 368.46 & 2303.67 & 2153.21 & 3415.69 \\
$\Pi_{T}^{*}$ & 24539.8 & 20867.35 & 15007.46 & 14536.45 & 17174.3 \\
\hline & & & & & \\
\end{tabular}

\section{NuMERICAL EXAMPLE}

To illustrate our formulated model, we consider the values of the parameters as follows: $c_{c}=\$ 10 ; p_{r c}=\$ 1$; $\rho_{1}=1 ; \theta_{\max }=100 ; \gamma=1.3 ; p_{s 0}=\$ 25 ; a_{1}=300 ; a_{2}=5 ; a_{3}=2.25 ; a_{4}=4 ; a_{5}=2 ; b_{1}=325 ; b_{2}=5.2 ; b_{5}=1$; $\xi=\$ 12 ; \eta=\$ 12 ; k_{1}=0.8 ; \phi=0.85 ; \tau_{m}=1.75 ; \tau_{r}=6 ; \tau_{c}=2.5 ; \tau_{s}=1.5$. For this hypothetical data set, the eigenvalues of the $\mathrm{HM}$ of the profit function $\Pi_{T}$ be $-15.868,-13.799,-10.8526,-2.24039$ under $\mathrm{CP}$ at the values of the decision variables listed in the following Table 2 . Hence the profit $\Pi_{T}$ is maximum, and the optimal values of the decision variables are stored, and each players' profits are calculated as in Table 2. Under the strategyCL, the eigenvalues of the HMs corresponding to the profit functions $\Pi_{m}$ and $\Pi_{r}$ are $-16.2986,-3.74321$, and $-13.2361,-8.76393$, respectively. Hence, the optimality condition holds, and the functions $\Pi_{m}$ and $\Pi_{r}$ are maximum at the respective values of decision variables. Again for the strategy SL, the HMs corresponding to the profit functions $\Pi_{m}$ and $\Pi_{r}$ are negative definite as the eigenvalues of the Matrices are $-16.3788,-3.75069$, and $-13.2361,-8.76393$ respectively. Hence the profit functions are maximum. Similarly, under the strategies CS and NS, the HMs of the profit functions are negative definite. Hence, the functions are maximum at the optimal values of the decision variables.

From Table 2, some results can be concluded as follows:

(1) The leader manufacturer holds a higher profit for all the studied cases.

(2) The best fit game, according to the profits for the players, is listed in the following:

(a) The manufacturer and the retailer gain most under CP.

(b) The Collector and the supplier make their maximum profit under the NG.

(3) The PE is maximum in CP. Therefore the customers will get the best effort from the retailer if the players participate centrally.

(4) The customers have to pay the lowest under the case: CS Nash. Although, in this game, the level of PE is also the smallest respect for the other games.

(5) Under the CP, the manufacturer can produce most eco-friendly products (i.e., the level of GI is maximum under this game). As a result, the environment-conscious players will be keener to join in the CP.

\section{Sensitivity ANALYSiS}

Now, the sensitivity of the parameters $\rho_{1}, \xi, \eta, a_{2}$ and $b_{2}$ are analyzed on the players' profits, TPTs, and the decision variables. Initially, the values of the parameters are set as below: $c_{c}=10 ; \phi=0.85 ; p_{r c}=1 ; \theta_{\max }=100$; $\gamma=1.3 ; k_{1}=0.8 ; p_{s 0}=25 ; \tau_{m}=1.75 ; \tau_{c}=2.5 ; \tau_{s}=1.5 ; \tau_{r}=6 ; a_{1}=300 ; a_{3}=2.25 ; a_{4}=4 ; b_{1}=325 ; a_{5}=2 ;$ $b_{5}=1 ; \rho_{1}=1$. In the figure 2 , it can be observed that the curve representing the TPT under CP is placed 
topmost and then the TPTs under NG, SL game, CL game one by one and lastly, the curve representing the TPT of CS nash placed at the bottom. Therefore the sequence of the TPTs under each parameters' effect is as $\Pi_{c} \geq \Pi_{T}^{n} \geq \Pi_{T}^{s l} \geq \Pi_{T}^{c l} \geq \Pi_{T}^{c s n}$, where $n, s l, c l, c s n$ stands for NG, SL game, CL game, and CS Nash respectively.

Whenever $\rho_{1}$ increases, the rate of recyclable waste materials increases, and the number of fresh materials decreases with an increment of $\rho_{1}$. Consequently, all the decision variables increase with the increment of $\rho_{1}$ except $p_{d}$ (see Tab. 3). As a result, the demand rates and the profit margins of the chain increase. Also, the profits of the manufacturer and the retailer increase with the increment of $\rho_{1}$ (see Figs. 2a, 3a, and 4a).

In the Figures $2 \mathrm{~b}, 3 \mathrm{~b}$ and $4 \mathrm{~b}$, the horizontal axis represents the values of $\xi$ and the vertical axis represents the profits. It can observed that, the curves are in downward direction with the increment of the value of horizontal axis. Hence the cost parameter of the GI level $(\xi)$ is inversely proportional to the profits of the manufacturer, retailer, and SCN since all the decision variables except $p_{d}$, and the demand rates decrease with the increment of this parameter.

In Figure 3c, the curve representing the profits of the manufacturer remains fixed with the change of $\eta$. Because, it is considered in the numerical example that the impact of PE is not as much as of GI on the customer's mind. Furthermore, the retailer provides PE. Therefore, the decision variables of the manufacturer (see Tab. 3) change a niggling and also is the same for his profit (see Fig. 3c). Again, in Figures 2c and 4c, the curves represent the retailer's and total chain profit respectively and it can be observed that they go gradually in the downward direction with the increment of the cost parameter of the PE $(\eta)$. Hence, if $\eta$ increases then all the profits decreases. Again, in Figure 2d and 2e, the curves are downward direction with the increment of the values of horizontal axis. Hence, the TPT of the SCN decreases for higher values of the own channel price sensitivity parameters $\left(a_{2}\right.$ and $\left.b_{2}\right)$, because all the decision variables and the demand rates diminish with the increments of those parameters (see Tab. 3). It can be concluded similarly for the sensitivity of these two parameters on the manufacturer's and the retailer's profit (see Figs. 3d, 4d, 3e and 4e).

\subsection{Total profit}

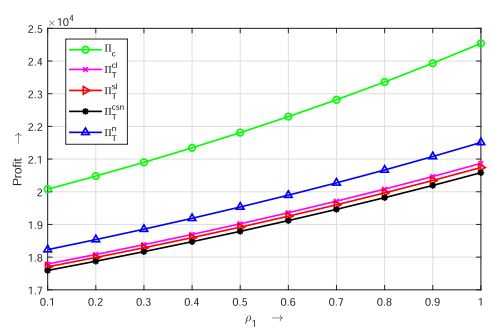

(a)

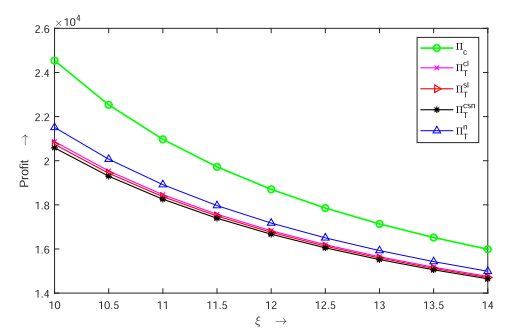

(b)

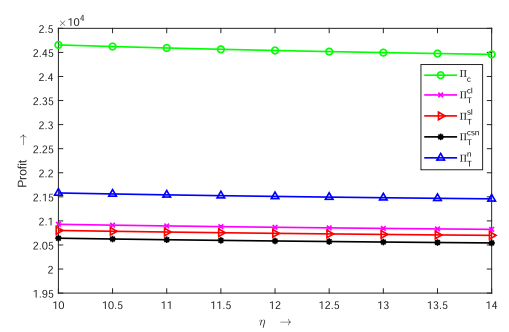

(c)

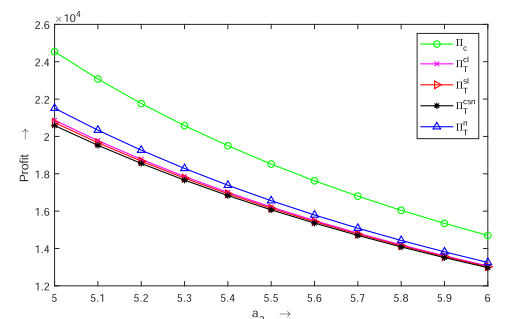

(d)



(e)

Figure 2. Effect of the parameters on the TPT of the SCN. (a) TPT versus $\rho_{1}$. (b) TPT versus $\xi$. (c) TPT versus $\eta$. (d) TPT versus $a_{2}$. (e) TPT versus $b_{2}$. 


\subsection{Manufacturer's profit}

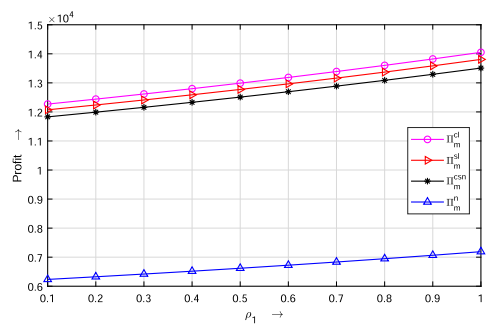

(a)



(b)

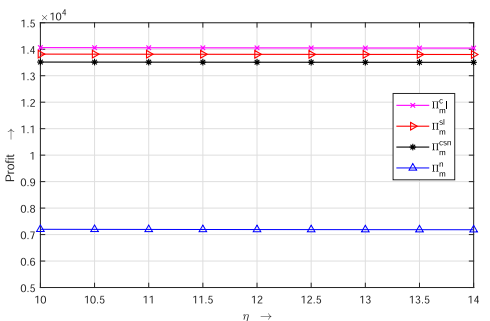

(c)



(d)

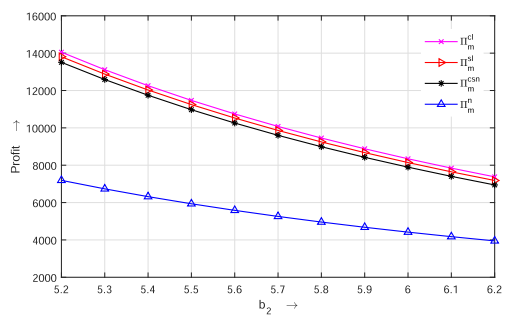

(e)

FiguRE 3. Effect of the parameters on the Manufacturer's profit. (a) Manufacturer profit versus $\rho_{1}$. (b) Manufacturer's profit versus $\xi$. (c) Manufacturer's profit versus $\eta$. (d) Manufacturer's profit versus $a_{2}$. (e) Manufacturer's profit versus $b_{2}$.

\subsection{Retailer's profit}

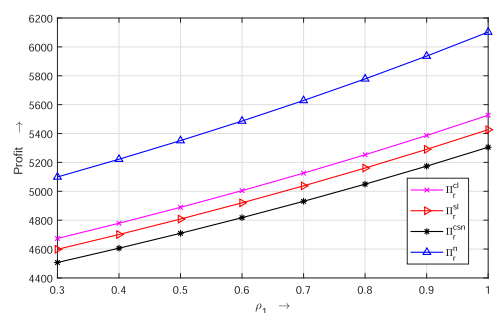

(a)

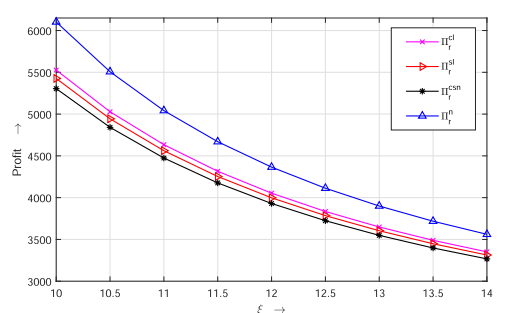

(b)

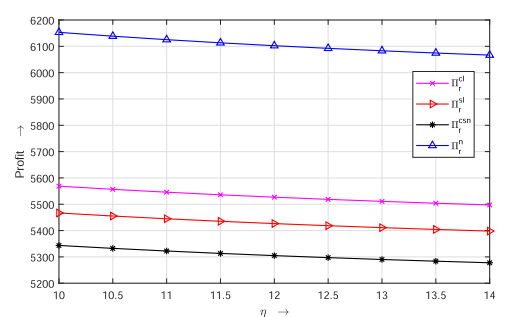

(c)

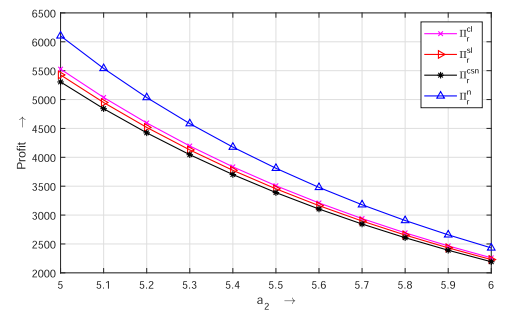

(d)

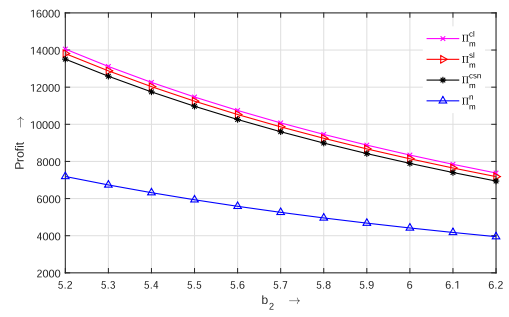

(e)

FigURE 4. Effect of the parameters on the Retailer's profit. (a) Retailer profit versus $\rho_{1}$. (b) Retailer's profit versus $\xi$. (c) Retailer's profit versus $\eta$. (d) Retailer's profit versus $a_{2}$. (e) Retailer's profit versus $b_{2}$. 


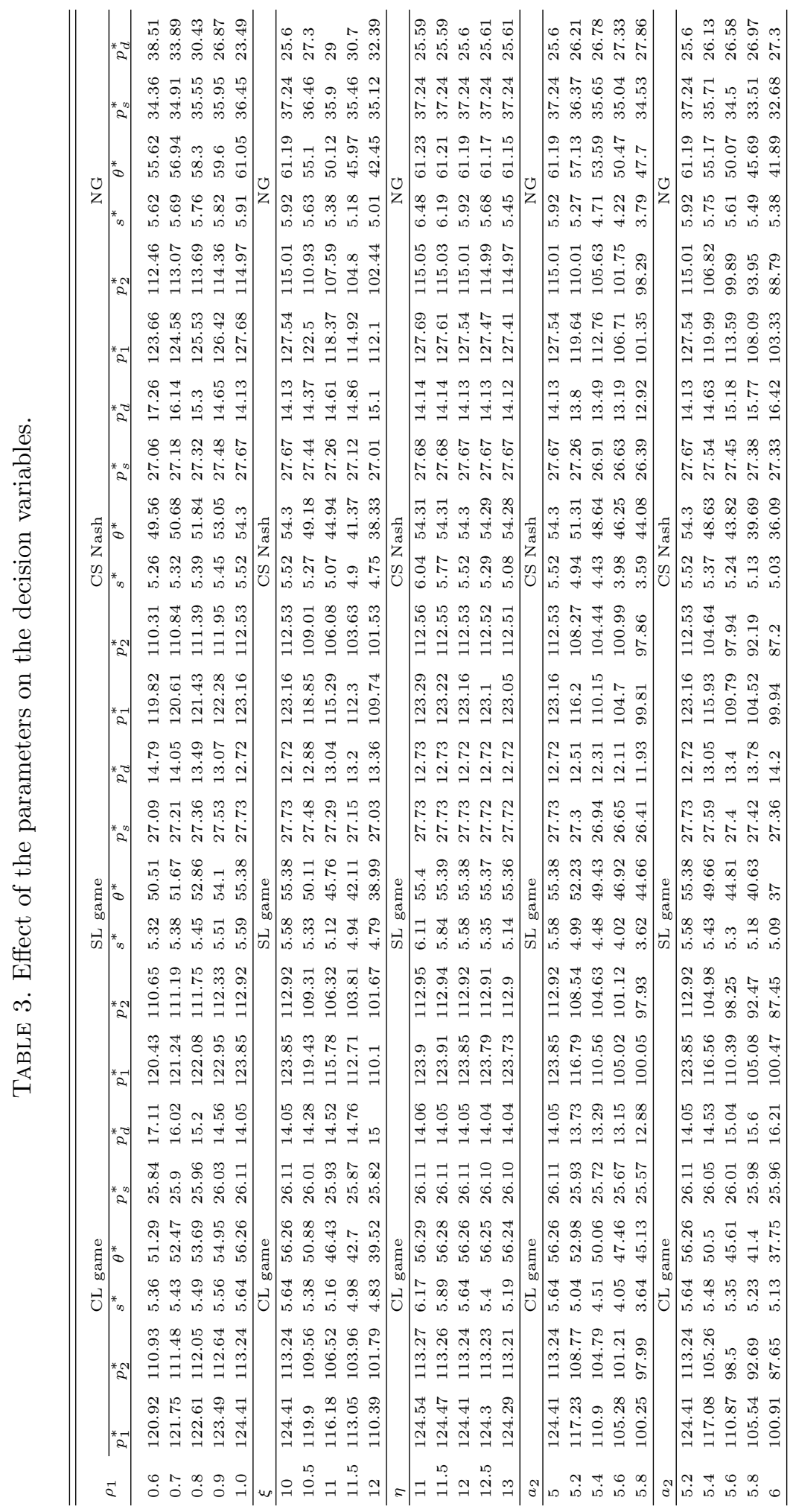




\section{MANAGERIAL INSIGHTS}

In this section, we draw some important insights that will be more profitable for a supply chain similar to our proposed model with sensitivity analysis. The manufacturer should improve products with their GI so that the rate of recyclability of the product increases and the profits of the channel. But the cost coefficient of the GI

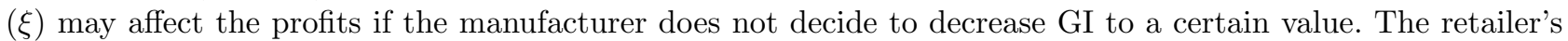
$\mathrm{PE}$ has a positive effect on the demand rate to help the retailer gain more. But if the cost coefficient of PE $(\eta)$ decreases, the retailer should decide to increase the PE so that the demand rate of his channel increases. Besides, if the pricing sensitivity of the demand rates $\left(a_{1}\right.$ and $\left.b_{1}\right)$ increases, then each players' profit decrease. Therefore they should decrease the values of $a_{1}$ and $b_{1}$ so that they make more profits. Under CP, the manufacturer can impose higher GI on the product, and the retailer can provide greater PE to the customers than the other strategies. From the SCN's point of view, CP is the best policy to make the most profit than any other strategy. Therefore, if the members work collaboratively, then they can make the highest profit. But the collector and the supplier make their maximum profit under NG comparing to other strategies.

\section{Conclusion}

In this paper, environmental sustainability has been studied by collecting waste from the end customers and recycling them. In the shortage of recyclable waste material, the manufacturer buys the extra fresh raw materials from the supplier to meet the demands. A dual-channel CLP SCN has been considered, where the collector sells the collected recyclable wastes to the manufacturer, and the manufacturer sells his finished products through retail channels as well as direct channels. The retailer provides PE to the customers, and the manufacturer imposes the greenness on the products while manufacturing. This model has described the CP and MS games, where the Stackelberg game separates the study in the four directions, such as CL game, SL game, CS Nash, and NG structures. The significant outcomes of the study are as follows:

(1) The rate of recyclable waste materials $(\beta)$ is directly proportional to the GI level $(\theta)$. Whenever the rate of recyclable waste increases, then the demand of the corresponding materials increases. Consequently, it increases the profits for each player.

(2) If the cost coefficient of GI $(\xi)$ decreases, then the manufacturer become keener to increase the level of GI. Consequently, the demand rates will increase and the profits of the members will increase with the increment of the level of GI.

(3) If the cost coefficient of PE $(\eta)$ decreases, then the retailer will try to increase the level of PE so that he can attract more customers to be a part of the retail channel. Consequently, the demands of the retail channel increase with the decrement of $\eta$. As a result, a higher PE level always gives more profit to the retailer in all cases.

(4) If the pricing sensitivity of demand rate increases, then it decreases the demand of the corresponding channel as well as the profits of the members. Therefore, it can be concluded that pricing sensitivity is inversely proportional to the profit of each player.

(5) Under MS settings, the manufacturer's and the retailer's profit is utmost under the CL game, whereas the collector and the supplier are more benefitted in the NG.

(6) The TPT of the SCN under CP is higher than all other game strategies.

Several research works can be done related to this direction. In this paper, the demand function is a deterministic type, but it can be straightaway modified by considering stochastic demand. This study considers a SCN with a single manufacturer and single retailer, but investigating a DC SCN with multiple-manufacturer or multiple-retailer might make the collaboration problem more difficult. Also, one can consider different types of coordination between the players to obtain higher profit. 
Acknowledgements. The authors would like to express their gratitude to the editors and referees for their valuable suggestions and corrections to enhance the clarity of the present article. The second author also acknowledges the Council of Scientific \& Industrial Research, Government of India for financial assistance.

\section{REFERENCES}

[1] M. Bhattacharyya and S.S. Sana, A mathematical model on eco-friendly manufacturing system under probabilistic demand. RAIRO:OR 53 (2018) 1899-1913.

[2] K. Cao, P. He and Z. Liu, Production and pricing decisions in a dual-channel supply chain under remanufacturing subsidy policy and carbon tax policy. J. Oper. Res. Soc. 71 (2019) 1199-1215.

[3] L.E. Cárdenas-Barrón and S.S. Sana, Multi-item EOQ inventory model in a two-layer supply chain while demand varies with promotional effort. Appl. Math. Model. 39 (2015) 6725-6737.

[4] X. Chen, X. Wang and X. Jiang, The impact of power structure on the retail service supply chain with an O2O mixed channel. J. Oper. Res. Soc. 67 (2016) 294-301.

[5] W.-Y.K. Chiang, D. Chhajed and J.D. Hess, Direct marketing, indirect profits: a strategic analysis of dual-channel supply-chain design. Manage. Sci. 49 (2003) 1-20.

[6] K.E. Clow and D. Baack, Integrated Advertising, Promotion, and Marketing Communications. Pearson Education Ltd, New York (2014).

[7] J. Gao, Z. Xiao, H. Wei and G. Zhou, Active or passive? Sustainable manufacturing in the direct-channel green supply chain: a perspective of two types of green product designs. Transp. Res. Part D: Transp. Environ. 65 (2018) 332-354.

[8] N. Georgantzis and C. Brunner, Promotional Effort. Springer Science+Business Media, New York (2016).

[9] P.D. Giovanni, A joint maximization incentive in closed-loop supply chains with competing retailers: the case of spent-battery recycling. Eur. J. Oper. Res. 268 (2018) 128-147.

[10] B.C. Giri and S.K. Dey, Game theoretic analysis of a closed-loop supply chain with backup supplier under dual channel recycling. Comput. Ind. Eng. 129 (2019) 179-191.

[11] M. Grimmer and T. Bingham, Company environmental performance and consumer purchase intentions. J. Bus. Res. 66 (2013) 1945-1953.

[12] C. He and H. Zhou, A retailer promotion policy model in a manufacturer Stackelberg dual-channel green supply chain. Proc. CIRP 83 (2019) $722-727$.

[13] W. Huang and J.M. Swaminathan, Introduction of a second channel: implications for pricing and profits. Eur. J. Oper. Res. 194 (2009) 258-279.

[14] H. Jafari, S.R. Hejazi and M. Rasti-Barzoki, Sustainable development by waste recycling under a three-echelon supply chain: a game-theoretic approach. J. Cleaner Prod. 142 (2017) 2252-2261.

[15] H. Jafari, S.R. Hejazi and M. Rasti-Barzoki, Sustainable development by waste recycling under a three-echelon supply chain: a game-theoretic approach. J. Cleaner Prod. 142 (2019) 2252-2261.

[16] R. Karim and K. Nakade, Modelling a one retailerone manufacturer supply chain system considering environmental sustainability and disruption. To appear in: Int. J. Syst. Sci. Oper. Logistics (2020). DOI: 10.1080/23302674.2020.1756006.

[17] B. Li, M. Zhu, Y. Jiang and Z. Li, Pricing policies of a competitive dual-channel green supply chain. J. Cleaner Prod. 112 (2016) 2029-2042.

[18] C. Mondal, B.C. Giri and T. Maiti, Pricing and greening strategies for a dual-channel closed-loop green supply chain. Flexible Serv. Manuf. J. 32 (2019) 724-761.

[19] D. Navinchandra, Steps Toward Environmentally Compatible Product and Process Design: A Case For Green Engineering. Carnegie Mellon University, Pennsylvania, United States (1990) 1-6.

[20] A. Ranjan and J.K. Jha, Pricing and coordination strategies of a dual-channel supply chain considering green quality and sales effort. J. Cleaner Prod. 218 (2019) 409-424.

[21] A. Roy, S.S. Sana and K. Chaudhuri, A joint venturing of single supplier and single retailer under variable price, promotional effort and service level. Pac. Sci. Rev. B: Humanities Soc. Sci. 1 (2015) 8-14.

[22] K. Salimifard and R. Raeesi, A green routing problem: optimising $\mathrm{CO}_{2}$ emissions and costs from a bi-fuel vehicle fleet. Int. J. Adv. Oper. Manage. 6 (2014) 27-57.

[23] A. Sarkar and B. Pal, Competitive pricing strategies of multi channel supply chain under direct servicing by the manufacturer. RAIRO:OR 55 (2021) S1849-S1873.

[24] R. Shankar, S. Bhattacharyya and A. Choudhary, A decision model for a strategic closed-loop supply chain to reclaim End-ofLife Vehicles. Int. J. Prod. Econ. 195 (2018) 273-286.

[25] C.-L. Shi, W. Geng and J.-B. Sheu, Integrating dual-channel closed-loop supply chains: forward, reverse or neither? To appear in: J. Oper. Res. Soc. (2020). DOI: 10.1080/01605682.2020.1745700.

[26] T. Stuckia, M. Woerterb, S. Arvanitisb, M. Penederc and C. Rammerd, How different policy instruments affect green product innovation: a differentiated perspective. Energy Policy 114 (2018) 245-261.

[27] L. Wang and Q. Song, Pricing policies for dual-channel supply chain with green investment and sales effort under uncertain demand. Math. Comput. Simul. 171 (2020) 79-93.

[28] J. Wei, W. Chen and G. Liu, How manufacturer's integration strategies affect closed-loop supply chain performance. To appear in: Int. J. Prod. Res. (2020). DOI: 10.1080/00207543.2020.1762016. 
[29] C.Y. Wong, C.W.Y. Wong and S. Boon-itt, Effects of green supply chain integration and green innovation on environmental and cost performance. Int. J. Prod. Res. 58 (2020) 4589-4609.

[30] X. Xie, J. Huo and H. Zou, Green process innovation, green product innovation, and corporate financial performance: a content analysis method. J. Bus. Res. 101 (2019) 697-706.

[31] L. Xu, C. Wang, Z. Miao and J. Chen, Governmental subsidy policies and supply chain decisions with carbon emission limit and consumers environmental awareness. RAIRO:OR 53 (2019) 1675-1689.

[32] H. Yang, L. Chu and H. Wan, Advertising and pricing policies in a two-Echelon supply chain with a capital-constrained retailer. RAIRO:OR 53 (2019) 1331-1342.

[33] A. Yenipazarli, To collaborate or not to collaborate: prompting upstream eco-efficient innovation in a supply chain. Eur. J. Oper. Res. 260 (2017) 571-587.

[34] Y. Yuan, J. Yang, Y. Li and W. Li, Necessary conditions for coordination of dual-channel closed-loop supply chain. Technol. Forecasting Soc. Change 151 (2020) 119823. 